\title{
Comparative evaluation of tomographic and biometric characteristics in bilateral keratoconus patients with unilateral corneal Vogt's striae: a contralateral eye study
}

This article was published in the following Dove Press journal:

Clinical Ophthalmology

\author{
Mohamad-Reza Sedaghat ${ }^{\prime}$ \\ Farshad Askarizadeh ${ }^{2}$ \\ Foroozan Narooie-Noori ${ }^{3}$ \\ Tahereh Rakhshandadi ${ }^{3}$ \\ Hadi Ostadi-moghadam ${ }^{2}$ \\ Sattar Rajabi ${ }^{2}$ \\ 'Cornea Research Center, Khatam-Al- \\ Anbia Hospital, Mashhad University \\ of Medical Sciences, Mashhad, \\ Iran; ${ }^{2}$ Refractive Errors Research \\ Center, Mashhad University of \\ Medical Sciences, Mashhad, Iran; \\ ${ }^{3}$ Department of Optometry, School \\ of Rehabilitation Sciences, Zahedan \\ University of Medical Sciences, \\ Zahedan, Iran
}

\begin{abstract}
Purpose: The aim of this study was to evaluate and compare tomographic and biometric characteristics measured by the corneal tomography and ocular biometry in bilateral keratoconus $(\mathrm{KCN})$ patients with and without corneal Vogt's striae.

Methods: Ninety-two eyes of 46 subjects with a reliable diagnosis of bilateral KCN with unilateral Vogt's striae were enrolled in this cross-sectional contralateral eye study. In addition to refraction (calculated by vectorial analysis) and visual acuity, corneal tomographic measurements were obtained by the Pentacam (Scheimpflug-based anterior segment tomography). Also, ocular biometric characteristics were evaluated using the Ocuscan ${ }^{\circledR} \mathrm{RxP}$ (ultrasound biometer). The KCN eyes were categorized into two groups, including eyes with Vogt's striae and eyes without Vogt's striae.
\end{abstract}

Results: Our results showed significant differences in the sphere, cylinder, spherical equivalent, J0, corrected and uncorrected distance visual acuity, flat, steep and maximum keratometry, anterior chamber depth (ACD), and central corneal thickness (CCT) between the two groups (all $P<0.001$ ). The eyes without Vogt's striae had a shorter ACD measured by the Pentacam and biometer. There were no differences in axial length (AL) and vitreous length (VL) between the two groups (all $P>0.05$ ). Also, there was poor agreement between the measurements of the Pentacam and ultrasound biometer for ACD in the study groups.

Conclusion: Corneal tomographic and ocular biometric measurements showed significant differences between KCN eyes with and without Vogt's striae except for AL and VL. These differences should be noticed in clinical evaluations and treatment of $\mathrm{KCN}$ patients.

Keywords: cornea, keratoconus, corneal tomography, ocular biometry, Vogt's striae

\section{Introduction}

Keratoconus $(\mathrm{KCN})$ is an asymmetric, bilateral condition which may have a unilateral onset. This non-inflammatory corneal ectasia is progressive and associated with stromal thinning, irregular astigmatism, and apical corneal bulge. ${ }^{1,2}$ There are numerous reports about the prevalence of $\mathrm{KCN}$, ranging from $0.05 \%$ to $2.5 \% .^{2-4} \mathrm{KCN}$ impairs vision and affects the vision-related quality of life, which continues to deteriorate over time. ${ }^{5,6}$ With progression of KCN, biomicroscopic signs such as stromal thinning, corneal scars, Fleischer's ring, and Vogt's striae may be seen on slit-lamp biomicroscopy. ${ }^{1,7,8}$ One of the typical biomicroscopic signs of $\mathrm{KCN}$ is the Vogt's striae, which may be unilateral or bilateral. ${ }^{8,9}$ The Vogt's striae are fine vertical lines generated by compaction of the Descemet's membrane and deep stroma that are parallel to the axis of the cone and
Correspondence: Farshad Askarizade Department of Optometry, School of Paramedical Sciences, Mashhad University of Medical Sciences, Vakil-abad Street, Mashhad 9I77948964, Iran

Tel +98912 l43996 |

Fax +98 215 54I 8080

Email asgarifarshad@yahoo.com
Clinical Ophthalmology 2018:12 1383-1390 Dovepress in http://dx,doi.org/10.214710PTH.S169266 (c) (1) (-) 2018 Sedaghat et al. This work is published and licensed by Dove Medical Press Limited. The full terms of this license are available at https://www.dovepress.com/terms.php cC) and incorporate the Creative Commons Attribution - Non Commercial (unported, v3.0) License (http://creativecommons.org/licenses/by-nc/3.0/). By accessing the work you hereby accept the Terms. Non-commercial uses of the work are permitted without any further permission from Dove Medical Press Limited, provided the work is properly attributed. For permission for commercial use of this work, please see paragraphs 4.2 and 5 of our Terms (https://www.dovepress.com/terms.php). 
vanish when physical compression is applied to the cornea. ${ }^{9}$ The Vogt's striae are also known as dark and light banding patterns and stress lines. ${ }^{9,10}$ Although this condition is defined as vertical lines, ${ }^{9}$ there are some reports of unilateral or bilateral horizontal Vogt's striae in KCN eyes. ${ }^{11,12}$

The Collaborative Longitudinal Evaluation of $\mathrm{KCN}$ (CLEK) study showed $34 \%$ of $\mathrm{KCN}$ patients have unilateral Vogt's striae, while the striae are bilateral in $30 \%$ of the cases. ${ }^{8}$

A few studies have assessed the associations of Vogt's striae with refractive and corneal alterations in $\mathrm{KCN}$ eyes. ${ }^{9,13}$ Hollingsworth and Efron evaluated the characteristics of corneal Vogt's striae in keratoconic eyes using in vivo confocal microscopy ${ }^{9}$ and Mocan et al compared refraction and keratometry in KCN patients with and without Vogt's striae. ${ }^{13}$

On the other hand, in novel refractive and cataract procedures [phakic intraocular lens (IOL) implantation, phacoemulsification with IOL implantation], accurate measurement of the axial length (AL) is essential to attain acceptable refractive outcomes. In addition to AL, anterior chamber depth (ACD), lens thickness (LT), vitreous length (VL), and corneal curvature are the most important factors for calculating a precise implantable IOL power. ${ }^{14-16}$ Moreover, the majority of the mentioned components in addition to corneal parameters (corneal thickness and curvature) are valuable for assessment of follow-up and treatment consequences of KCN patients. ${ }^{17-19}$ Different studies about biometric characteristics of KCN eyes have used different measurement devices without considering the Vogt's striae..$^{20,21}$

The purpose of this study was to evaluate and compare corneal tomographic and ocular biometric measurements using a Scheimpflug-based tomography and a contact ultrasound biometer in $\mathrm{KCN}$ eyes with and without Vogt's striae.

\section{Methods}

This cross-sectional comparative-descriptive study was carried out at Sedaghat Eye Clinic, Mashhad, Iran from February to June 2017. All participants in this study were residents of Mashhad with the same ethnicity.

According to the Declaration of Helsinki, written informed consent was obtained from all participants and the study was approved by the Institutional Review Board/Ethics Committee of Mashhad University of Medical Sciences.

The sampling was done based on pilot study and crosssectional nature of the study. Therefore, 92 eyes of 46 subjects with a reliable diagnosis of bilateral $\mathrm{KCN}$ with unilateral Vogt's striae were enrolled in this study.

All the participants underwent a comprehensive ophthalmic examination, including a full patient history, uncorrected distance visual acuity (UDVA), corrected distance visual acuity (CDVA), manifest and cycloplegic refraction by autorefractokeratometer (KR-1, Topcon Corporation, Tokyo, Japan), irregularity of the retinoscopic reflex with scissoring in both eyes, non-contact computerized tonometry (Topcon Corporation), slit-lamp biomicroscopy, funduscopy, Scheimpflug-based anterior segment tomography (Pentacam HR, Oculus, Optikgerate GmbH, Wetzlar, Germany) and placido disc-based corneal topography (TMS4, Tomey, Enlargen, Germany).

All measurements were performed between 04:00 pm and 06:00 pm by one skilled optometrist (FA).

The inclusion criterion for the $\mathrm{KCN}$ eyes was a reliable diagnosis of KCN made by an experienced corneal refractive surgeon based on slit-lamp signs, as well as corneal topography/tomography and pachymetry maps. The inclusion criteria for KCN patients were at least one clinical sign on slit lamp biomicroscopy (Vogt's striae, Fleischer's ring, apical thinning, etc.), ${ }^{1,8}$ topographic signs of KCN (skewed asymmetric bow-tie, inferior steepening, etc.), ${ }^{22}$ tomographic presentations of KCN (abnormal elevation maps, abnormal pachymetry maps, KCN detection by Belin/Ambrosio Enhanced Ectasia Display III, etc.). ${ }^{23}$

The exclusion criteria in all the groups were previous eye surgery, history of corneal cross linking, corneal scars, vascularization, inflammation, opacity, history of herpetic keratitis, severe dry eye, contact lens use during the last three weeks before the examination, cataract, glaucoma or glaucoma suspect, intraocular pressure-lowering treatment, pregnancy, lactation, and underlying autoimmune or systemic diseases. Eyes diagnosed as $\mathrm{KCN}$ suspect or pellucid marginal degeneration were excluded from the study as well.

Considering the mentioned criteria, we included 46 patients with bilateral KCN but unilateral Vogt's striae in this study. The $\mathrm{KCN}$ eyes were categorized into two groups, including KCN eyes with Vogt's striae and KCN eyes without Vogt's striae. Then, the following corneal measurements were recorded using the Pentacam: corneal power (flat, steep and maximum keratometry), ACD, and central corneal thickness (CCT).

The same experienced optometrist obtained all corneal imaging and biometric measurements in a consistent manner based on the manufacturers' instructions and previous studies. ${ }^{24-27}$ Measurements based on quality state and index provided by Pentacam were accepted and erroneous acquisitions were repeated after 5 minutes. The mechanism of the Pentacam has been described in previous studies. ${ }^{24}$ Although studies have shown acceptable repeatability and reproducibility of the Pentacam imaging for the normal cornea, ${ }^{25,26}$ 
it should be considered that Pentacam measurements are variable in patients with $\mathrm{KCN} .{ }^{27}$ Therefore, three measurements were done at 5-minute intervals with each device (the Pentacam and ultrasound biometry) for each patient and mean value of the three measurements was used for statistical analysis. Moreover, the AL, ACD, LT, and VL were measured using the Alcon Ocuscan ${ }^{\circledR} \mathrm{RxP}$ (Alcon, Fort Worth, TX, USA) ultrasound biometer. The Ocuscan ${ }^{\circledR}$ $\mathrm{RxP}$ is an ophthalmic contact ultrasound device developed to measure ocular biometry in an easy to use format. This device is reliable in clinical use and we also applied this system for A-scan biometry. ${ }^{28}$ Biometry was performed at the end of the measurements to avoid its probable influence on the other imaging modalities due to corneal flattening after applanation with the ultrasound probe. The mechanism of this device and its measurement method has been discussed in other studies. ${ }^{28}$ Before performing A-scan ultrasonography, in order to anesthetize the cornea, one drop of tetracaine 0.5\% (Anestocaine; Sina Darou, Iran) was instilled in each eye. Since there was no study to assess the repeatability of ultrasound biometry in the $\mathrm{KCN}$ eyes, the measurements were repeated three times (10 results for each measurement) per eye and the mean value of the measurements was recorded. If the SD of a measurement was greater than $\pm 0.12 \mathrm{~mm}$ for $\mathrm{AL}$, the measurement was rejected and the procedure was repeated until the SD was in the range of $\pm 0.12 \mathrm{~mm}$. If the participants suffered from poor fixation, low visual acuity, and high myopia, which made the results unreliable, the measurements were repeated to achieve acceptable results. To control the effect of accommodation while measuring the lens thickness, the patients were requested to look at a target placed at six meters with their fellow eye. ACD, LT, $\mathrm{AL}$, and VL were noted in each eye.

The manufacturer's representative checked the calibration of the devices before the study and we did not change or adjust the default settings for corneal tomography and ocular biometry.

Power vector analysis was performed to compare refraction between the two study groups. The results of spherocylindrical refraction were converted to vectors expressed by three dioptric powers: $\mathrm{M}, \mathrm{J} 0$, and $\mathrm{J} 45[\mathrm{M}=\mathrm{S}+(\mathrm{C} / 2)$, $\mathrm{J} 0=-\mathrm{C} / 2 \cos (2 \alpha), \mathrm{J} 45=-\mathrm{C} / 2 \sin (2 \alpha)]$, where $\mathrm{M}$ was equal to the spherical equivalent of the given refractive error, and $\mathrm{J} 0$ and $\mathrm{J} 45$ were the 2 Jackson cross cylinder equivalents to the conventional cylinder. Cycloplegic refraction was recorded in the conventional manner (sphere, cylinder, and axis) and then converted to the coordinates of the power vector as described by Thibos and Horner. ${ }^{29}$

\section{Statistical analysis}

Statistical analysis was performed using Statistical Package for Social Sciences (SPSS) software version 22 (IBM Corporation, Armonk, NY, USA) and MedCalc Software version 15.8.X86 (MedCalc Software Bvba, Ostend, Belgium). Normal distribution of the parameters was assessed using the Kolmogorov-Smirnov test. A paired sample $t$-test was run to compare the parameters with a normal distribution while a Wilcoxon signed rank test was used to compare non-parametric parameters. Pearson correlation coefficient was run for correlative analyses. In order to study the agreement between the measurements made by devices, the Bland and Altman method was used. The 95\% limits of agreement (LoA) (mean difference $\pm 1.96 \mathrm{SD}$ [SD]), which defines the range within which most differences between measurements by the two methods will lie, were calculated. $P$-values $<0.05$ were considered significant.

\section{Results}

We assessed 46 bilateral $\mathrm{KCN}$ patients [28 males $(60.87 \%)$ and 18 females $(39.13 \%)]$ in this study. The mean age of the participants was $26.61 \pm 6.99$ years (range: $18-45$ years).

There were significant differences in the refractive outcomes between the two groups (all $P<0.001$ ) except for J45 $(P<0.131)$. Some basic characteristics of the subjects are shown in Table 1. It should be mentioned that the outcomes of cycloplegic refraction were used in this study.

Contralateral comparison of biometric characteristics (measured by ultrasound biometry) between $\mathrm{KCN}$ eyes with and without Vogt's striae are presented in Table 2. The results of paired sample $t$-test showed the eyes without Vogt's striae had shorter $\operatorname{ACD}(P=0.001)$ and greater LT $(P=0.010)$, but there were no differences in $\mathrm{AL}(P=0.307)$ and $\mathrm{VL}(P=0.756)$ between the two groups.

The results of the paired sample $t$-test showed that there were significant differences in the corneal tomographic characteristics between two study groups. Comparison of Scheimpflug-based corneal tomographic measurements between $\mathrm{KCN}$ eyes with and without Vogt's striae are presented in Table 3.

In $\mathrm{KCN}$ eyes with and without Vogt's striae, investigations of the correlations between tomographic and biometric measurements are shown in Table 4.

It should be mentioned that the Pentacam measures "internal" ACD (aqueous depth from the posterior corneal surface), whereas Ocuscan ${ }^{\circledR}$ RxP evaluates the ACD from the anterior surface of the cornea (external ACD). In order to calculate the external ACD for Pentacam results, we added 
Table I Contralateral comparison of basic parameters between keratoconus eyes with and without Vogt's striae

\begin{tabular}{|c|c|c|c|c|c|c|}
\hline \multirow[t]{2}{*}{ Parameter } & \multicolumn{2}{|c|}{ With Vogt's striae } & \multicolumn{2}{|c|}{ Without Vogt's striae } & \multirow{2}{*}{$\begin{array}{l}\text { Mean } \\
\text { difference }\end{array}$} & \multirow[t]{2}{*}{$P$-value } \\
\hline & Mean \pm SD & Range & Mean \pm SD & Range & & \\
\hline Sph (D) & $-3.28 \pm 2.67$ & -8.75 to $+\mathrm{I} .75$ & $-0.98 \pm 1.90$ & $-7.25-1.50$ & $-2.29 \pm 2.68$ & $<0.00 \mathrm{I}^{\mathrm{a}}$ \\
\hline Cyl (D) & $-5.03 \pm 2.10$ & -8.75 to -1.75 & $-2.52 \pm 1.90$ & $-8.25-0.00$ & $-2.5 \mathrm{I} \pm 2.28$ & $<0.00 \mathbf{I}^{\mathrm{a}}$ \\
\hline SE (D) & $-5.97 \pm 3.50$ & -16.62 to -0.12 & $-2.24 \pm 2.43$ & $-10.12-0.88$ & $-3.73 \pm 3.37$ & $<0.00 \mathrm{I}^{\mathrm{a}}$ \\
\hline J0 (D) & $1.30 \pm 1.94$ & $-4.00-7.93$ & $0.30 \pm 0.10$ & $-2.07-2.62$ & $0.10 \pm 1.62$ & $<0.00 \mathrm{I}^{\mathrm{b}}$ \\
\hline $\mathrm{J} 45$ (D) & $0.32 \pm 1.83$ & $-3.7 \mid-3.13$ & $-0.19 \pm 1.12$ & $-3.25-2.62$ & $0.55 \pm 2.62$ & $<\left.0.13\right|^{a}$ \\
\hline UDVA (Log MAR) & $0.68 \pm 0.45$ & $0.00-1.60$ & $0.4 I \pm 0.46$ & $0.00-1.60$ & $0.26 \pm 0.36$ & $<0.00 \mathrm{I}^{\mathrm{a}}$ \\
\hline CDVA (Log MAR) & $0.3 I \pm 0.30$ & $0.00-1.00$ & $0.09 \pm 0.11$ & $0.00-0.40$ & $0.23 \pm 0.26$ & $<0.00 \mathrm{I}^{\mathrm{a}}$ \\
\hline
\end{tabular}

Notes: a Wilcoxon signed ranks test. ${ }^{b}$ Paired-samples $t$-test. Bold values are significant.

Abbreviations: Sph, sphere; Cyl, cylinder; SE, spherical equivalent; J0, Jackson cross cylinder; axes at $0^{\circ}$ and $90^{\circ}$; J45, Jackson cross cylinder; axes at $45^{\circ}$ and I35 $35^{\circ}$ UDVA, uncorrected distance visual acuity; CDVA, corrected distance visual acuity; D, diopter; Log MAR, logarithm of the minimum angle of resolution.

the CCT and ACD values together and then performed the desired statistical analyses.

As shown in Table 5, there was no correlation between the two imaging devices in terms of external ACD in two study groups $(P>0.05)$. Also, 95\% LoA were determined for better comparison of the degree of agreement between the two methods.

According to Table 5 and Figure 1, there was no reasonable agreement between the measurements of the Pentacam and ultrasound biometer for external $\mathrm{ACD}$ in $\mathrm{KCN}$ eyes with and without Vogt's striae.

According to the staging scheme in CLEK study, ${ }^{30}$ keratoconic eyes were classified into three groups based on keratometry of steepest corneal meridian: mild ( $<45$ diopters $[\mathrm{D}])$, moderate $(45-52 \mathrm{D})$ and severe $(>52 \mathrm{D})$. In selected eyes with Vogt's striae, $2(4 \%), 10(20 \%)$, and 38 (76\%) of eyes were in mild, moderate, and severe groups, respectively, whereas in KCN eyes without Vogt's striae, 11 (22\%), 28 $(56 \%)$, and $11(22 \%)$ of eyes were in mild, moderate, and severe groups, respectively.

\section{Discussion}

In order to evaluate $\mathrm{KCN}$ eyes and to achieve the best refractive outcome after refractive (phakic IOL implantation, intracorneal ring implantation) and cataract surgery in these patients, tomographic and biometrics characteristics should be determined precisely. ${ }^{14-19}$ Meanwhile, determination of ocular biometric characteristics in patients with $\mathrm{KCN}$, as the most common form of corneal ectatic disorders, ${ }^{1,2}$ is necessary to achieve the best post keratoplasty refractive outcomes, and to estimate the treatment sequels and follow-up of these patients. ${ }^{17}$

On the other hand, Vogt's striae or stress lines, which may be visualized on slit lamp biomicroscopy, are fine vertical lines in the deep stroma that could be considered an important clinical indicator of $\mathrm{KCN}^{.7-10,13}$

To the best of our knowledge, this is the first contralateral eye study to investigate and compare corneal tomographic and ocular biometric characteristics of bilateral $\mathrm{KCN}$ patients with and without Vogt's striae.

Our results revealed that $\mathrm{KCN}$ eyes with Vogt's striae had higher values of sphere, spherical equivalent, cylinder, and J0 than those without Vogt's striae. Additionally, UDVA and CDVA were worse in eyes with Vogt's striae than eyes without these lines. Using Scheimpflug-based tomography, we found that flat, steep and maximum keratometry were higher in eyes with Vogt's striae. Also, CCT measurements revealed a thinner cornea in eyes with Vogt's striae. The above-mentioned differences have an essential value in the clinical practice. Indeed, the presence of Vogt's striae in keratoconic eyes seems to be an indicator of significant alterations in refractive and tomographic measurements.

Table 2 Contralateral comparison of biometric characteristics between keratoconus eyes with and without Vogt's striae

\begin{tabular}{|c|c|c|c|c|c|c|}
\hline \multirow[t]{2}{*}{ Parameter } & \multicolumn{2}{|c|}{ With Vogt's striae } & \multicolumn{2}{|c|}{ Without Vogt's striae } & \multirow{2}{*}{$\begin{array}{l}\text { Mean } \\
\text { difference }\end{array}$} & \multirow[t]{2}{*}{$P$-value ${ }^{a}$} \\
\hline & Mean \pm SD & Range & Mean \pm SD & Range & & \\
\hline $\mathrm{AL}(\mathrm{mm})$ & $23.49 \pm 0.87$ & $21.28-25.29$ & $23.44 \pm 0.89$ & $20.77-25.14$ & $0.05 \pm 0.30$ & 0.307 \\
\hline $\mathrm{ACD}(\mathrm{mm})$ & $3.70 \pm 0.39$ & $2.84-4.66$ & $3.60 \pm 0.36$ & $2.98-4.57$ & $0.10 \pm 0.20$ & 0.001 \\
\hline $\mathrm{VL}(\mathrm{mm})$ & $16.14 \pm 0.83$ & $13.94-18.26$ & $16.15 \pm 0.84$ & $13.90-17.92$ & $-0.0 \mathrm{I} \pm 0.25$ & 0.756 \\
\hline $\mathrm{LT}(\mathrm{mm})$ & $3.65 \pm 0.26$ & $3.20-4.18$ & $3.69 \pm 0.26$ & $3.18-4.18$ & $-0.04 \pm 0.10$ & 0.010 \\
\hline
\end{tabular}

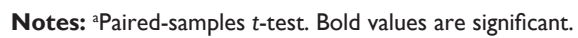

Abbreviations: $A L$, axial length; $A C D$, anterior chamber depth; $V L$, vitreous length; $L T$, lens thickness. 
Table 3 Contralateral comparison of tomographic characteristics between keratoconus eyes with and without Vogt's striae

\begin{tabular}{|c|c|c|c|c|c|c|}
\hline \multirow[t]{2}{*}{ Parameter } & \multicolumn{2}{|c|}{ With Vogt's striae } & \multicolumn{2}{|c|}{ Without Vogt's striae } & \multirow{2}{*}{$\begin{array}{l}\text { Mean } \\
\text { difference }\end{array}$} & \multirow[t]{2}{*}{$P$-value } \\
\hline & Mean \pm SD & Range & Mean \pm SD & Range & & \\
\hline $\mathrm{KI}$ front $(\mathrm{D})$ & $48.58 \pm 4.75$ & $42.20-65.70$ & $44.83 \pm 2.40$ & $39.30-51.10$ & $3.74 \pm 4.50$ & $<0.00 I^{\mathrm{a}}$ \\
\hline $\mathrm{K} 2$ front (D) & $53.14 \pm 5.23$ & $43.80-71.20$ & $47.45 \pm 3.34$ & $40.80-56.30$ & $5.70 \pm 5.52$ & $<0.00 \mathrm{I}^{\mathrm{b}}$ \\
\hline KI back (D) & $-7.23 \pm 0.90$ & -10.20 to -5.70 & $-6.31 \pm 1.02$ & -7.60 to -0.40 & $-0.92 \pm 1.20$ & $<0.00 \mathrm{I}^{\mathrm{b}}$ \\
\hline K2 back (D) & $-7.63 \pm 3.06$ & $-10.60-11.80$ & $-7.03 \pm 0.65$ & -8.80 to -5.70 & $-0.60 \pm 3.14$ & $<0.00 \mathrm{I}^{\mathrm{b}}$ \\
\hline Kmax (D) & $59.21 \pm 6.07$ & $48.10-77.00$ & $51.40 \pm 5.42$ & $44.10-67.40$ & $7.8 I \pm 5.74$ & $<0.00 I^{\mathrm{a}}$ \\
\hline ССТ $(\mu \mathrm{m})$ & $457.20 \pm 3.25$ & $369.00-537.00$ & $487.13 \pm 35.80$ & $409.00-553.00$ & $-29.94 \pm 25.57$ & $<0.00 I^{\mathrm{a}}$ \\
\hline $\mathrm{ACD}(\mathrm{mm})$ & $3.56 \pm 0.36$ & $2.79-4.47$ & $3.4 I \pm 0.40$ & $2.28-4.24$ & $0.15 \pm 0.25$ & $<0.00 \mathrm{I}^{\mathrm{a}}$ \\
\hline
\end{tabular}

Notes: All parameters were measured by Pentacam. aPaired-samples $t$-test. ${ }^{b}$ Wilcoxon signed ranks test. Bold values are significant.

Abbreviations: KI, flat keratometry; K2, steep keratometry; Kmax, maximum keratometry; CCT, central corneal thickness; ACD, anterior chamber depth (internal).

The results of ultrasound biometry showed no difference in AL and VL between KCN eyes with and without Vogt's striae. However, ultrasound biometry showed that ACD in KCN eyes with Vogt's striae was $0.1 \mathrm{~mm}$ deeper than those without Vogt's striae. According to the literature, the ACD is deeper in $\mathrm{KCN}$ eyes in comparison to normal eyes, which may be due to morphological alterations in the cornea as formerly explained by some authors. ${ }^{2}$ However, our results showed that a deeper ACD can be seen at the presence of Vogt's striae. Considering the effect of preoperative ACD on IOL power calculation, ${ }^{31,32}$ it seems that it is better to use new generation IOL formulas for IOL power calculation in $\mathrm{KCN}$ patients with Vogt's striae. Furthermore, LT was different between KCN eyes with and without Vogt's striae and was

Table 4 Correlation coefficients of refractive, tomographic, and biometric characteristics of keratoconus eyes with and without Vogt's striae

\begin{tabular}{|c|c|c|c|c|c|c|c|c|}
\hline \multirow[t]{2}{*}{ Parameter } & \multicolumn{2}{|c|}{$A L^{a}(\mathrm{~mm})$} & \multicolumn{2}{|c|}{$A C D^{a}(\mathrm{~mm})$} & \multicolumn{2}{|c|}{$V^{a}(\mathbf{m m})$} & \multicolumn{2}{|c|}{$\mathbf{L T}^{\mathbf{a}}(\mathrm{mm})$} \\
\hline & $P$-value ${ }^{c}$ & $r$ & $P$-value ${ }^{c}$ & $r$ & $P$-value ${ }^{c}$ & $r$ & $P$-value ${ }^{c}$ & $r$ \\
\hline \multicolumn{9}{|l|}{$\mathrm{AL}^{\mathrm{a}}(\mathrm{mm})$} \\
\hline With Vogt's striae & - & - & 0.019 & 0.345 & $<0.001$ & 0.928 & 0.333 & -0.146 \\
\hline Without Vogt's striae & - & - & 0.002 & 0.436 & $<0.001$ & 0.935 & 0.197 & -0.194 \\
\hline \multicolumn{9}{|l|}{$\mathrm{ACD}^{\mathrm{a}}(\mathrm{mm})$} \\
\hline With Vogt's striae & - & - & - & - & 0.636 & 0.072 & $<0.001$ & -0.506 \\
\hline Without Vogt's striae & - & - & - & - & 0.081 & -0.260 & $<0.001$ & -0.582 \\
\hline \multicolumn{9}{|l|}{$\mathrm{VL}^{\mathrm{a}}(\mathrm{mm})$} \\
\hline With Vogt's striae & - & - & - & - & - & - & 0.136 & -0.224 \\
\hline Without Vogt's striae & - & - & - & - & - & - & 0.081 & -0.260 \\
\hline \multicolumn{9}{|l|}{$\mathrm{CCT}^{\mathrm{b}}(\mu \mathrm{m})$} \\
\hline With Vogt's striae & 0.450 & 0.114 & 0.692 & 0.060 & $0.46 \mathrm{I}$ & 0.111 & 0.739 & -0.050 \\
\hline Without Vogt's striae & 0.953 & 0.009 & 0.723 & -0.054 & 0.643 & 0.070 & 0.718 & -0.055 \\
\hline \multicolumn{9}{|l|}{$\mathrm{KI} \mathrm{I}^{\mathrm{b}}(\mathrm{D})$} \\
\hline With Vogt's striae & 0.062 & -0.280 & 0.110 & 0.239 & 0.010 & -0.376 & 0.944 & -0.011 \\
\hline Without Vogt's striae & 0.051 & -0.291 & $0.8 \mathrm{II}$ & 0.036 & 0.055 & -0.285 & 0.821 & 0.034 \\
\hline \multicolumn{9}{|l|}{$\mathrm{K} 2^{\mathrm{b}}(\mathrm{D})$} \\
\hline With Vogt's striae & 0.155 & -0.213 & 0.368 & 0.136 & 0.053 & -0.288 & 0.619 & 0.075 \\
\hline Without Vogt's striae & 0.250 & -0.173 & 0.520 & -0.097 & 0.342 & -0.143 & 0.546 & 0.091 \\
\hline \multicolumn{9}{|l|}{$\mathrm{Kmax}^{\mathrm{b}}(\mathrm{D})$} \\
\hline With Vogt's striae & 0.214 & -0.187 & 0.083 & 0.259 & 0.116 & -0.235 & 0.193 & -0.195 \\
\hline Without Vogt's striae & 0.479 & -0.107 & 0.873 & -0.024 & 0.446 & -0.115 & 0.552 & 0.090 \\
\hline \multicolumn{9}{|l|}{ SE (D) } \\
\hline With Vogt's striae & 0.302 & -0.156 & 0.120 & -0.232 & 0.497 & -0.103 & 0.303 & 0.156 \\
\hline Without Vogt's striae & 0.941 & 0.011 & 0.497 & 0.103 & 0.497 & -0.103 & 0.452 & -0.114 \\
\hline
\end{tabular}

Notes: aMeasured by ultrasound biometer. ${ }^{b}$ Measured by Pentacam. 'Pearson correlation coefficient analysis. Bold values are significant.

Abbreviations: AL, axial length; ACD, anterior chamber depth; VL, vitreous length; LT, lens thickness; CCT, central corneal thickness; KI, flat keratometry; K2, steep keratometry; Kmax, maximum keratometry; SE, spherical equivalent. 
Table 5 Pentacam and ultrasound biometer measurements for anterior chamber depth of keratoconus eyes with and without Vogt's striae

\begin{tabular}{|c|c|c|c|c|c|c|c|c|}
\hline \multirow{2}{*}{$\begin{array}{l}\text { Parameter } \\
\text { Device }\end{array}$} & \multirow{2}{*}{$\frac{\text { Pentacam }}{\text { Mean } \pm \text { SD }}$} & \multirow{2}{*}{$\frac{\text { Ultrasound }}{\text { Mean } \pm \text { SD }}$} & \multicolumn{2}{|l|}{ Difference } & \multicolumn{2}{|c|}{ Correlation } & \multicolumn{2}{|c|}{ 95\% LoA } \\
\hline & & & $\Delta$ & $P$-value ${ }^{a}$ & $r$ & $P$-value & Lower & Upper \\
\hline \multicolumn{9}{|l|}{$\overline{A C D}(\mathrm{~mm})$} \\
\hline With Vogt's striae & $4.0 \mathrm{I} \pm 0.36$ & $3.70 \pm 0.39$ & $-0.3 I \pm 0.53$ & 0.0002 & -0.005 & 0.974 & -0.72 & 1.35 \\
\hline Without Vogt's striae & $3.90 \pm 0.39$ & $3.60 \pm 0.36$ & $-0.30 \pm 0.53$ & 0.0004 & 0.006 & 0.968 & -0.74 & 1.33 \\
\hline
\end{tabular}

Notes: aPaired-samples $t$-test. Bold values are significant.

Abbreviations: ACD, anterior chamber depth; $\Delta$, mean difference; $r$, Pearson correlation coefficient; LoA, limit of agreement.
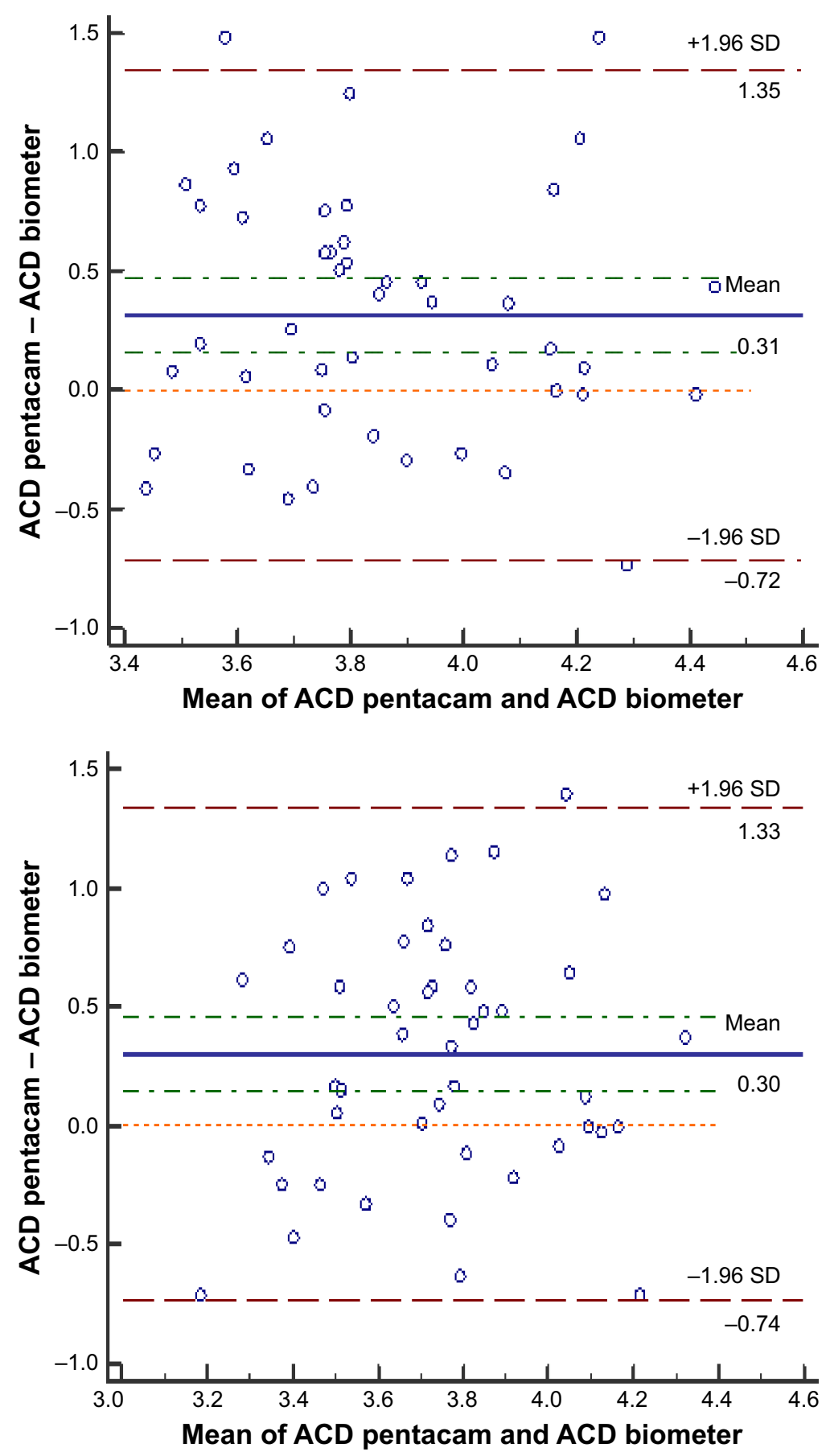

Figure I Bland-Altman plots of the ACD measured by the Pentacam against ultrasound biometer in KCN eyes with Vogt's striae (top) and without Vogt's striae (bottom). The middle line in each figure is the mean difference of the values and the lines on the sides represent the upper and lower $95 \%$ LoA (mean difference \pm I.96 SD). Abbreviations: ACD, anterior chamber depth; KCN, keratoconus; LoA, limits of agreement. 
thicker in KCN eyes with Vogt's striae. This difference was only statistically significant $(0.04 \mathrm{~mm}, P=0.010)$ and further studies are needed to assess the clinical importance of it.

We studied possible correlations between biometric results in KCN eyes with and without Vogt's striae. We found an intermediate correlation between $\mathrm{AL}$ and $\mathrm{ACD}$ in $\mathrm{KCN}$ eyes with and without Vogt's striae. Moreover, AL had a strong correlation with VL in $\mathrm{KCN}$ eyes with and without Vogt's striae. Also, CCT, steep keratometry, maximum keratometry, and spherical equivalent were not correlated with AL, ACD, LT, and VL in either study group. Meanwhile, there was a poor correlation between flat keratometry and VL in KCN eyes with Vogt's striae. What is certain is that the interpretation of the relationships between different ocular components is a very complex issue and is not the main focus of our study.

Moreover, we evaluated the agreement between the Pentacam and ultrasound biometer for measurement of ACD but failed to find a reasonable agreement between them in the study groups. Also, the results of the present study showed a wide LoA for ACD measurements on Pentacam and ultrasound biometer in either study group. To our knowledge, this is the first study to assess the agreement among ACD measurements in $\mathrm{KCN}$ eyes with and without Vogt's striae using Pentacam and ultrasound biometer. It can be concluded that one should be cautious interpreting ACD values obtained from Pentacam and ultrasound biometer in eyes with $\mathrm{KCN}$.

Mocan et al found an association between Vogt's striae with refraction and corneal topography in $\mathrm{KCN}$. They reported significant differences in the refractive components and steepest keratometry between patients with and without corneal Vogt's striae. ${ }^{13}$ Our results are consistent with their findings, but it should be borne in mind that Mocan et al assessed refraction and corneal curvature and did not assess corneal tomography and ocular biometry, while we assessed clinical, tomographic, and ocular biometric parameters in a contralateral eye study in $\mathrm{KCN}$ patients with and without Vogt's striae. Also, they did not use a power vector to analyze the refractive astigmatism.

In another study, Hollingsworth and Efron studied the correlation between the arrangement of the Vogt's striae in the stroma and the steepest corneal curvature of the cornea using corneal topography. ${ }^{9}$ They did not compare the clinical and subclinical findings between $\mathrm{KCN}$ patients with and without Vogt's striae.

It is noteworthy that no study has evaluated and compared tomographic and biometric characteristics of $\mathrm{KCN}$ eyes with and without Vogt's striae; hence, we are unable to compare the results of the present study with other published articles. However, a large body of literature has described the differences between $\mathrm{KCN}$ and normal eyes.

Although our study is remarkable for complete assessment and comparison of tomographic and biometric characteristics in $\mathrm{KCN}$ eyes with and without Vogt's striae, performing this study without using in vivo confocal microscopy should be noted as a limitation. Another limitation that can be mentioned for the present study is that considering the study design and asymmetric nature of $\mathrm{KCN}$, the majority of the selected eyes with Vogt's striae had severe $\mathrm{KCN}$.

Although this contralateral eye study has substantial advantages, we had a limitation for matching KCN severity between two study groups. The same limitation was reported by Mocan et al in their study. ${ }^{13}$ Based on steepest keratometry values, $22 \%$ of KCN eyes without Vogt's striae had severe $\mathrm{KCN}$ in the current study. Interestingly, we found that $\mathrm{KCN}$ eyes with clinical evidence of Vogt's striae had worse corneal tomographic findings. Classification of $\mathrm{KCN}$ severity only based on keratometry values may seem rational but this staging scheme may lead us astray from a clinical point of view. The results of the present study cast doubt on the validity of keratometry-based classification of $\mathrm{KCN}$ severity. Therefore, it is recommended to design a study to compare clinical characteristics of KCN eyes with and without Vogt's striae in patients who have similar steep keratometry values.

\section{Conclusion}

In summary, this comparative-descriptive study of keratoconic eyes with and without Vogt's striae appear to suggest that in ophthalmic evaluation, attention should be paid to the clinical characteristics of KCN eyes with Vogt's striae. In this case of evidence-based medicine, the results showed the association of Vogt's striae with KCN severity. Vogt's striae have been reported to be an indicator of $\mathrm{KCN}^{7}$ but our results showed different clinical manifestations in eyes with and without Vogt's striae. Moreover, Pentacam and ultrasound biometry could not be used interchangeably for ACD measurement in KCN eyes. This important clinical finding should be considered in phakic IOL implantation to improve the visual outcomes of $\mathrm{KCN}$ eyes. ${ }^{18,19}$ It is critical to note that the present study did not aim to show the causal relationship between Vogt's striae and clinical characteristics of $\mathrm{KCN}$ eyes, but to explain the association of the stromal Vogt's striae with tomographic and biometric alterations in $\mathrm{KCN}$ eyes. The results of our study can be used in clinical assessment, monitoring, and treatment of $\mathrm{KCN}$ patients with and without Vogt's striae. In conclusion, we found that the 
presence of Vogt's striae is associated with deterioration in refractive, tomographic, and biometric findings.

\section{Acknowledgment}

The authors would like to thank all the willing participants in this study, without whose involvement this research would not have been possible.

\section{Disclosure}

The authors report no conflicts of interest in this work.

\section{References}

1. Rabinowitz YS, Keratoconus RYS. Keratoconus. Surv Ophthalmol. 1998;42(4):297-319.

2. Romero-Jiménez M, Santodomingo-Rubido J, Wolffsohn JS. Keratoconus: a review. Cont Lens Anterior Eye. 2010;33(4):157-166.

3. Hashemi H, Beiranvand A, Khabazkhoob M, et al. Prevalence of keratoconus in a population-based study in Shahroud. Cornea. 2013; 32(11):1441-1445.

4. Jonas JB, Nangia V, Matin A, Kulkarni M, Bhojwani K. Prevalence and associations of keratoconus in rural maharashtra in central India: the central India eye and medical study. Am J Ophthalmol. 2009;148(5): $760-765$.

5. Aydin Kurna S, Altun A, Gencaga T, Akkaya S, Sengor T. Vision related quality of life in patients with keratoconus. J Ophthalmol. 2014;2014(4):1-7.

6. Kymes SM, Walline JJ, Zadnik K, Sterling J, Gordon MO; Collaborative longitudinal evaluation of keratoconus study group. Changes in the quality-of-life of people with keratoconus. American J Ophthalmol. 2008;145(4):611-617.

7. Grieve K, Ghoubay D, Georgeon C, et al. Stromal striae: a new insight into corneal physiology and mechanics. Sci Rep. 2017;7(1):13584.

8. Zadnik K, Barr JT, Edrington TB, et al. Baseline findings in the Collaborative Longitudinal Evaluation of Keratoconus (CLEK) study. Invest Ophthalmol Vis Sci. 1998;39(13):2537-2546.

9. Hollingsworth JG, Efron N. Observations of banding patterns (Vogt striae) in keratoconus: a confocal microscopy study. Cornea. 2005;24(2): $162-166$.

10. Somodi S, Hahnel C, Slowik C, Richter A, Weiss DG, Guthoff R. Confocal in vivo microscopy and confocal laser-scanning fluorescence microscopy in keratoconus. Ger J Ophthalmol. 1996;5(6):518-525.

11. Chung SH, Kim EK. Keratoconus with unilateral horizontal stress lines. Cornea. 2005;24(7):890.

12. Güngör IU, Beden U, Sönmez B. Bilateral horizontal Vogt's striae in keratoconus. Clin Ophthalmol. 2008;2(3):653-655.

13. Mocan MC, Yilmaz PT, Irkec M, Orhan M. The significance of Vogt's striae in keratoconus as evaluated by in vivo confocal microscopy. Clin Exp Ophthalmol. 2008;36(4):329-334.

14. Olsen T. Calculation of intraocular lens power: a review. Acta Ophthalmol Scand. 2007;85(5):472-485.
15. Bozorg S, Pineda R. Cataract and keratoconus: minimizing complications in intraocular lens calculations. Semin Ophthalmol. 2014;29(5-6): 376-379.

16. Guerra MG, Silva AMM, Marques SHM, et al. Phakic intraocular lens implantation: refractive outcome and safety in patients with anterior chamber depth between 2.8 and 3.0 versus $\geq 3.0 \mathrm{~mm}$. Ophthalmic Res. 2017;57(4):239-246.

17. Ernst BJ, Hsu HY. Keratoconus association with axial myopia: a prospective biometric study. Eye Contact Lens. 2011;37(1):2-5.

18. Esteve-Taboada JJ, Domínguez-Vicent A, Ferrer-Blasco T, Alfonso JF, Montés-Micó R. Posterior chamber phakic intraocular lenses to improve visual outcomes in keratoconus patients. J Cataract Refract Surg. 2017;43(1):115-130.

19. Kato N, Toda I, Hori-Komai Y, Sakai C, Arai H, Tsubota K. Phakic intraocular lens for keratoconus. Ophthalmology. 2011;118(3): 605.e2-605.e2.

20. Cinar Y, Cingü AK, Sahin M, et al. Comparison of optical versus ultrasonic biometry in keratoconic eyes. J Ophthalmol. 2013;2013: 481238.

21. Aurich H, Pham DT, Wirbelauer C. Biometric evaluation of keratoconic eyes with slit lamp-adapted optical coherence tomography. Cornea. 2011;30(1):56-59.

22. Matalia H, Swarup R. Imaging modalities in keratoconus. Indian $J$ Ophthalmol. 2013;61(8):394-400.

23. Belin MW, Ambrósio R. Scheimpflug imaging for keratoconus and ectatic disease. Indian J Ophthalmol. 2013;61(8):401-406.

24. Oliveira CM, Ribeiro C, Franco S. Corneal imaging with slit-scanning and Scheimpflug imaging techniques. Clin Exp Optom. 2011;94(1):33-42.

25. Lackner B, Schmidinger G, Pieh S, Funovics MA, Skorpik C. Repeatability and reproducibility of central corneal thickness measurement with Pentacam, Orbscan, and ultrasound. Optom Vis Sci. 2005;82(10): 892-899.

26. Mcalinden C, Khadka J, Pesudovs K. A comprehensive evaluation of the precision (repeatability and reproducibility) of the Oculus Pentacam HR. Invest Ophthalmol Vis Sci. 2011;52(10):7731-7737.

27. Vianna LM, Muñoz B, Hwang FS, Gupta A, Jun AS. Variability in Oculus Pentacam tomographer measurements in patients with keratoconus. Cornea. 2015;34(3):285-289.

28. Sohajda Z, Papp J, Berta A, Módis L. The comparative study of two recently developed A-scan devices: determination of central corneal thickness, anterior chamber depth and axial length. Acta Ophthalmol. 2008; 86(1):45-48.

29. Thibos LN, Horner D. Power vector analysis of the optical outcome of refractive surgery. J Cataract Refract Surg. 2001;27(1):80-85.

30. Zadnik K, Barr JT, Gordon MO, Edrington TB. Biomicroscopic signs and disease severity in keratoconus. Collaborative Longitudinal Evaluation of Keratoconus (CLEK) Study Group. Cornea. 1996;15(2): 139-146.

31. Miraftab M, Hashemi H, Fotouhi A, Khabazkhoob M, Rezvan F, Asgari S. Effect of anterior chamber depth on the choice of intraocular lens calculation formula in patients with normal axial length. Middle East Afr J Ophthalmol. 2014;21(4):307-311.

32. Jeong J, Song H, Lee JK, Chuck RS, Kwon JW. The effect of ocular biometric factors on the accuracy of various IOL power calculation formulas. BMC Ophthalmol. 2017;17(1):62.
Clinical Ophthalmology

\section{Publish your work in this journal}

Clinical Ophthalmology is an international, peer-reviewed journal covering all subspecialties within ophthalmology. Key topics include: Optometry; Visual science; Pharmacology and drug therapy in eye diseases; Basic Sciences; Primary and Secondary eye care; Patient Safety and Quality of Care Improvements. This journal is indexed on Submit your manuscript here: http://www.dovepress.com/clinical-ophthalmology-journal
Dovepress

PubMed Central and CAS, and is the official journal of The Society of Clinical Ophthalmology (SCO). The manuscript management system is completely online and includes a very quick and fair peer-review system, which is all easy to use. Visit http://www.dovepress.com/ testimonials.php to read real quotes from published authors. 